ISSN: 0213-3563

https://doi.org/10.14201/azafea2020222548

\title{
¿PUEDEN LOS ANIMALES SENTIR DOLOR? REFLEXIONES DESDE LA FENOMENOLOGÍA
}

Can Animals Feel Pain? Reflections from Phenomenology

Francisco Mujica

Institut supérieur de Philosophie - UCLouvain

Recibido: 18 de diciembre de 2018

Aceptado: 28 de noviembre de 2019

\section{RESUMEN}

La discusión filosófica relacionada con el dolor en los animals se ha centrado exclusivamente en el aspecto moral. En este artículo nos proponemos una pregunta más fundamental: si los animales son capaces de sentir dolor o no. Para abordar la cuestión mostraremos por qué la fenomenología parece ser la única corriente filosófica que puede tematizar del todo la experiencia del dolor. La fenomenología permite el descubrimiento filosófico del mundo de la vida, el descubrimiento filosófico de la conciencia como una forma de sentido, tanto como el descubrimiento filosófico del Ego como una estructura afectiva y sintiente. Con todo, las principales contribuciones de la fenomenología parecerían inútiles para entender el dolor animal. Teniendo esto en cuenta, concluiremos el artículo esbozando algunas intuiciones para desarrollar una explicación filosófica del dolor en los animales.

Palabras clave: animales; dolor; mundo; sentido; ego; afecto; sentimiento; fenomenología; humanismo; filosofía del dolor.

\section{ABSTRACT}

Philosophical discussion concerning animals' pain has focused exclusively on its moral feature. In this paper, we discuss a broader issue, namely, if animals can feel pain. To settle this matter, we will show why phenomenology seems to be the only philosophical perspective that can fully grasp 
pain experience. Phenomenology allowed the philosophical discovery of the lived-world, the philosophical exploration of conscience as a form of meaning, as well as the philosophical development of self as an affective and feeling structure. Nevertheless, the main philosophical contributions of phenomenology seem useless to grasp animal pain. That being said, we will conclude this paper by sketching some insights to develop a philosophical account of animal pain.

Key words: animals; pain; world; meaning; self; affection; feeling; phenomenology; humanism; philosophy of pain.

"La pregunta no es ¿pueden razonar?, ni ¿pueden hablar?, sino, ¿pueden sufrir?”

Bentham

\section{INTRODUCCIÓN: SOBRE LAS TRES CONTRIBUCIONES CAPITALES DE LA FENOMENOLOGÍA}

No resulta osado afirmar que la fenomenología constituye uno de los paradigmas intelectuales decisivos de nuestra época. Esta hipótesis puede ser sujeta a verificación cuando se evalúan las contribuciones diferenciales que la fenomenología realizó como consecuencia de su surgimiento y estabilización disciplinarios. Sin embargo, dichas contribuciones solo pueden sopesarse cuando se cuenta con aguda consciencia del estado de la filosofía en el momento de la aparición de la fenomenología. ¿Cuál era la situación socio-histórica de la filosofía y qué problemas había generado ella misma en su desenvolvimiento hacia el año 1900?

El período que va desde 1850 a 1900 es el primer periodo en la historia de Europa en que la filosofía desaparece. Más aún, en el momento en el que se prepara la obra de Husserl, no sólo la filosofía carecía de grandes exponentes disciplinarios ni de escuelas de pensamiento, sino que se asistía por aquel entonces a la instalación de un escepticismo socio-cultural en relación a la filosofía. No solo no había verdadera producción filosófica en la segunda mitad del siglo XIX, sino que el hombre occidental habia dejado de creer en la filosofía. Evidentemente que este sentimiento era una de las consecuencias del esplendor técnico-rendimental que experimentó Europa en el periodo de la Belle époque: "Europa se enriqueció; el mundo, vacío de sentido, se llenó de máquinas, se hizo cómodo. Esta fue la compensación: el utilismo sirvió de balancín al funámbulo europeo” (Ortega y Gasset, 1966, p. 509). 
Podemos ver, entonces, que la filosofía (la reflexión sobre los fundamentos, el pensamiento riguroso) parecía ya no entregar rendimiento, no otorgar prestaciones culturales, no tener función social: la máquina, su perenne operatividad y su inacabable rendimiento respondían ahora a todos los problemas del hombre europeo. No obstante, Husserl considera que esta situación es responsabilidad también de la filosofía misma. Husserl observa que las únicas filosofías de mediados del siglo XIX y comienzos del siglo XX (neokantismo, neo-hegelianismo) se extinguieron por hiper-intelectualización, por auto-fagocitación (Szilasi, 1973).

Nos encontramos entonces con el primer problema disciplinario generado por la filosofía en la época que Husserl constituye su proyecto filosófico. La conclusión kantiana sobre la inercia de la realidad (o sobre la inaccesibilidad a la realidad transfenomenal, al "noumeno") abre la puerta a una reificación de la realidad fenomenal. Es así como la escuela epistemológica del neo-kantismo a fines del siglo XIX (Natorp, Rickert, Cohen, Heimsholt) procederá a identificar fenómeno (aparecer, donación del objeto) con dato (magnitud objetiva, realidad fáctica) (Marcuse, 2003). En su apropiación positivista del proyecto idealista, la escuela neo-kantiana desdibuja la categoría sentido: el dato valdría ya solo por sí mismo - y no por su significación en una trama o contexto que le otorga su valor y significado fundamental (Housset, 2008).

A su vez, el hegelianismo y el neo-hegelianismo pretendieron hacer filosofía sin contacto ya con la realidad, obnubilada por su propia excedencia conceptual. No por casualidad afirma Gadamer (1993) que el método dialéctico de Hegel es, más bien, un enorme y cínico monólogo. La sobre-intelectualización hegeliana de la filosofía tuvo como consecuencia, asimismo, una sobre-intelectualización del pensamiento en general. En tanto el idealismo validó una metodología epistemológica que apostaba por una filosofía sin correlato externo, sin contacto firme con la realidad, la gran parte de las corrientes epistemológicas del siglo XIX (empirismo, mecanicismo, naturalismo, psicologismo), operan ya sin saber si su operación se trata de una descripción de la realidad o de una atribución intelectualista (Sartre, 1984).

La primera tarea filosófica de Husserl será entonces luchar contra el vicio del propio pensamiento, a saber: el intelectualismo, el espejismo del conceptualismo; el solipsismo como consecuencia de los excesos del pensamiento. Es por esto que el primer movimiento filosófico de Husserl será apuntar a una des-intelectualización de la filosofía con respecto a su estado disciplinario.

Para lograr des-intelectualizar a la filosofía, Husserl procederá a establecer cuál debe ser el objeto capital de la filosofía y del pensamiento en general. 
La respuesta de Husserl no deja lugar a dudas, a saber: la experiencia. Este es el sentido de la máxima fenomenológica "volver a las cosas mismas": la experiencia original del mundo cotidiano es la fuente de derecho de todo conocimiento y de todo saber (Husserl, 1995, \&24). No debe restringirse empero este concepto de experiencia a la utilización del mismo en el empirismo inglés. La experiencia científica es sólo una dimensión del concepto de experiencia originaria que Husserl busca restituir como referencia capital de todo análisis filosófico (Romano, 2010). Es por esto que Husserl subordina su análisis del concepto de experiencia a la forma que la experiencia adopta en la percepción (Merleau-Ponty, 2001).

Husserl considera que la percepción es la variante arquetípica de la experiencia dado que la percepción es la disposición fundamental de establecimiento de relación entre conciencia y mundo: la percepción inaugura no sólo el objeto a percibir, sino un horizonte para el despliegue de la percepción en particular, en general y para nuevas referencias y apariciones (Romano, 2010). Repárese en lo siguiente: sólo percibimos gracias a un trasfondo perceptivo, gracias a una trama de referencias reales, virtuales y potenciales que hacen posible la percepción. La percepción se revela así como una analogía perfecta de la experiencia: la experiencia solo está posibilitaba por una referencia implícita a nuevas experiencias, la experiencia supone un horizonte para su posibilidad, para su concreción y su interpretación que se sistematiza como un horizonte infinito de experiencias posibles (Gadamer, 1993).

El primer paso fundamental del proyecto filosófico de Husserl es redefinir el objeto fundamental de la filosofía. Ese objeto se llama experiencia, experiencia originaria del mundo vivido. Toda la filosofía desde Descartes vivió en la falsa precaución (casi paranoica) de que la realidad externa podía constituir una fuente de engaño y no de verdad. Ese problema se trató de solucionar subordinando el dato externo a la representación interna de la consciencia (Descartes, Kant, Berkeley, Fichte) o mostrando que en la falaz representación externa se disfrazaba una verdad escondida o subterránea (Leibniz, Schelling, Hegel) (Romano, 2010). Pues bien, Husserl muestra que "la experiencia en cuanto tal es la donación de un dato originario que excluye toda posibilidad de error. Y esto por definición, ya que el ser es lo que se manifiesta a la conciencia y la conciencia se agota en la donación del ser" (De Waelhens, 1953, p. 26; mi traducción). Ser y aparecer, donación y vivencia, fenómeno y esencia no están disociados, ya que la experiencia constituye una presencia originaria absolutamente indudable (Husserl, 1995: \&24). Es más, este postulado se ratifica en virtud de su indispensabilidad misma: si yo dudo 
de la presencia originaria de una experiencia particular dada en "carne y hueso aquí y ahora” es porque supongo que habrá otra efectivamente originaria que corregirá el darse defectuoso de la primera (Husserl, 1982). En último término, Husserl pone de manifiesto que la fuente de toda reflexión no está jamás dentro de la reflexión misma, sino en la experiencia primaria, en la evidencia ante-predicativa que la experiencia cotidiana entrega (De Waelhens, 1953) No por casualidad escribirá Michel Henry $(2001, \& 12)$ que el principio último de la fenomenología es: "tanto aparecer, tanto ser".

Llegamos así a la primera contribución capital de la fenomenología, a saber: la fenomenología le devuelve a la filosofía su referente externo, y ese referente externo lleva por nombre nada menos que mundo. La filosofía encuentra con Husserl, no sólo un referente externo, sino un borizonte -en la medida en que la estructura del mundo le otorga a la filosofía la posibilidad de mentar infinitos objetos (e infinitas perspectivas) (Housset, 2008).

Pero el descubrimiento husserliano del mundo, permite comprender, asimismo, la segunda gran contribución disciplinaria de la fenomenología.

El retorno husserliano a la experiencia originaria bajo la forma de percepción, abrió la puerta al descubrimiento filosófico del mundo. En este sentido, la noción de mundo lleva implícita en sí la segunda gran contribución de la fenomenología, a saber: la consciencia. No se trata aquí, sin embargo, de los conceptos de consciencia que han primado en filosofía desde Descartes. Contrariamente a una posición pasivo-sensualista (propia del empirismo), y también contra una concepción solipsista de la consciencia (propia del idealismo), Husserl (1995) considera que la consciencia no es una cosa (como hizo el naturalismo), sino que es sobre todo una operación. Esta operación se llama intencionalidad.

La pregunta de rigor es, entonces: ¿qué es intencionalidad? Si nos aproximamos a la noción de intencionalidad desde su sentido etimológico, descubrimos que: "La palabra intentio significa "dirigirse a". Toda vivencia, toda actitud anímica, se dirigen a algo. La percepción es, en cuanto tal, percepción "de algo", y lo mismo ocurre con la representación, el recuerdo, el juicio, la conjetura, la expectativa, la esperanza, el amor" (Szilasi, 1973, p. 32; cursivas y comillas del autor).

Una primera conclusión preliminar es la condición de principio que nos permite extraer la noción de intencionalidad, en tanto, todo acto de conciencia es, como se empeña en ratificar el mismo Husserl, por definición, intencional: "Una nota generalísima queda, empero como la válida para toda modalidad de 
la conciencia: el ser conciencia de algo. Este algo, el objeto intencional en cuanto tal de todo acto de conciencia, se halla presente a ésta como unidad idéntica de cambiantes modos de conciencia, ya sean noético-noemáticos, intuitivos o no intuitivos" (Husserl, 2004, p. 84; comillas del autor).

En virtud de lo anterior, encontramos una segunda propiedad elemental del principio de intencionalidad:

lo que caracteriza a la intencionalidad es la unidad de una acción de la conciencia con lo que se produce en ella (...) el "ver" y lo "visto" no pueden separarse el uno del otro (...) Del mismo modo, podemos decir: "Yo he deseado (he cumplido un acto de deseo), por eso deseo" (...) La acción de la conciencia es intentio, es decir: el producto está contenido en el acto productivo (Szilasi, 1973, pp. 40-41; cursivas y comillas del autor).

La explicitación de las propiedades fundamentales del principio de la intencionalidad de la conciencia nos permite ver que, no sólo debemos resaltar la cuestión de que todo hecho de conciencia se constituye como un complejo indisoluble entre un acto y a lo que ese acto refiere, sino que el principio de intencionalidad nos enseña que lo esencial de la conciencia es su estar volcada hacia la exterioridad, hacia objetos de conciencia trascendentes a ella misma:

De donde resulta naturalmente una distinción radical entre la conciencia y aquello de lo que se tiene conciencia. Cualquiera que sea el objeto de la conciencia (salvo en el caso de la conciencia reflexiva) está por principio fuera de la conciencia: es trascendente (Sartre, 1984, p. 183; cursivas del autor).

La determinación del estatuto del concepto de intencionalidad a la hora de estudiar la constitución nos ha permitido dilucidar cuál es la propiedad distintiva de la conciencia, a saber: ser conciencia de algo, estar referida a algo externo a ella. Se percibe ahora con total claridad la intrincación -la inseparabilidad-constitutiva de consciencia y mundo. No obstante, esta inseparabilidad no se debe a un mero afán disciplinario. Husserl subraya que esta conexión (que los excesos del idealismo y del cientificismo desdibujaron) es originaria y fundamental. Y no solo por la naturaleza originaria del dato ante-predicativo mismo -el proyecto filosófico de Husserl no es de carácter objetivista-, sino porque la consciencia es, antes que todo, una apertura al mundo que posibilita toda experiencia posible. Ortega y Gasset, con esa maravillosa claridad característica de su estilo, sintetiza el gran hallazgo de Husserl de forma magistral: 
Por lo visto, esa cosa que llamamos conciencia, es la más rara que hay en el universo, pues tal y como se nos presenta parece consistir en la conjunción, complexión o íntima perfecta unión de dos cosas totalmente distintas: mi acto de referirme a, y aquello a que me refiero. Y nótese bien toda la gravedad del caso: no es que nosotros a posteriori reconozcamos o descubramos la absoluta diferencia entre ambas cosas, sino que el hecho de conciencia consiste en que yo hallo ante mí algo como distinto y otro que yo. Esta mesa no es mi conciencia a buen seguro, mi conciencia ahora es ese "estar ante mí la mesa”; por tanto, la unidad inseparab1e de dos elementos tan absolutamente divergentes entre sí como son, por un lado, ese "estar ante mí”, por otro, la mesa (Ortega y Gasset 1963 , p. 63; cursivas y comillas del autor).

El descubrimiento husserliano de la intencionalidad de la consciencia no sólo da cuenta del hecho de que consciencia y mundo se suponen mutuamente en una correlación de donación y mención -el mundo se abre a las menciones de las consciencia y la consciencia menta el mundo en virtud de su donación-, sino que la intrincación entre conciencia y mundo implica que cada referencia o mención consciente es susceptible de sentido: cada referencia consciente al mundo implica-implícita o explícitamente (no es este el lugar para examinar la teoría husserliana del juicio)-, la afirmación de un posición tética, de aquí que, desde Husserl, toda mención o referencia contenga una significación fundamental, es decir, que toda referencia consciente representa una forma de sentido: cada mención objetual contiene, supone o da cuenta de una determinada inteligibilidad ideal y diferencial del objeto mentado. No por casualidad puede afirmar Husserl en el \&15 de su primera Investigación lógica: "Es en la significación donde se construye la relación al objeto. En consecuencia, emplear una expresión con sentido y referir con una expresión al objeto $(. .$.$) es una misma cosa". En conclusión: el mundo en su darse a$ la experiencia es una fuente de verdades potenciales y no de errores, ya que cada mención o referencia constituye una intención significativa; un acto de atribución de sentido al mundo en su donación (Levinas, 2004).

Debe destacarse que el develamiento fenomenológico de la consciencia como dotación de sentido nos conduce a la tercera contribución capital de la fenomenología.

La descripción husserliana de la percepción como arquetipo de la experiencia originaria, implica que la consciencia percipiente está ya-siempre involucrada y se ve interpelada (quiéralo o no) por el objeto percibido. Para la consciencia percipiente el sentido de lo percibido es ontológicamente inseparable del acto de percibirlo. Esto da cuenta que la estructura de la consciencia 
como acto de donación de sentido tiene como condición de posibilidad una capacidad implícita de auto-afección pre-intencional:

La consciencia no es nunca creación ex-nihilo, ella está siempre, más bien, motivada; ella remite a una sensibilidad que le da su impulso necesario (Montavont, 1999, p. 65; mi traducción).

Es así que, una vez descubierta la capacidad productora de sentido propia de la consciencia intencional, Husserl mostrará que toda forma de actividad intencional (percepción como sistema de síntesis activas) tiene como correlato necesario una forma de afección pre-intencional (o de síntesis pasiva):

el yo está siempre afectado (...) la afección del yo es obra de la pasividad, y esto en todos los niveles de la constitución. Al nivel de la esfera hylética inmanente, son las sensaciones, las pulsiones y los instintos que afectan al yo; al nivel de las esferas trascendentes, es la sedimentación de los objetos que el yo ha constituido en el transcurso de su génesis el factor afectante (Montavont, 1999, p. 72; mi traducción).

Llegamos así a la tercera gran contribución filosófica de la fenomenología. No es solo que la fenomenología descubra el mundo y el sentido; ella descubre, asimismo, la interioridad. Evidentemente que la interioridad como problema filosófico constituye un tema central del idealismo filosófico -particularmente en su raíz protestante (Bourgeois, 1995; Frank, 1997). Se trata allí, sin embargo, de una interioridad des-corporizada, a-sensorial, a-estesiológica. Con justa razón afirma entonces Levinas (2004) que la gran revolución filosófica desde Husserl es que "la sensibilidad es ya susceptible de razón": la fenomenología de Husserl es la primera corriente filosófica en tematizar y abordar la condición sintiente, afectante, padeciente propia de toda subjetividad (Levinas, 1967).

La explicitación fenomenológica de la condición afectante de toda subjetividad constituye una verdadera revolución, en tanto ella inaugura un campo de análisis inédito para filosofía: deseo, sensación, impresión, afecto, dolor serán finalmente susceptibles de tratamiento filosófico bajo el concepto de pasividad (Kühn, 1998). Pero la capacidad de sentir revelada por la fenomenología no sólo representa una revolución desde el punto de vista temático en filosofía, sino que también desde el punto de vista sistemático. En este sentido, se puede afirmar que el concepto de pasividad generará todo un nuevo aparataje conceptual en filosofía con vistas a enfocar y analizar la di- 
mensión pasiva de la subjetividad: cuerpo vivido (Leiblichkeit), carne (Leib), "Yo-puedo", kinestesia, motivación (Merleau-Ponty, 2001) serán algunos de los conceptos que derivarán del abordaje fenomenológico de la pasividad.

Toda vida es experiencia y toda experiencia es, por definición, mundana, significativa y afectante. Todo dolor es una experiencia, ergo, todo dolor ocurre en el mundo, todo dolor tiene un sentido atribuido y todo dolor implica un padecer. Mundo, sentido y afección constituyen las tres contribuciones filosóficas capitales de la fenomenología. A pesar de lo anterior, ¿puede sostenerse este postulado como principio analítico a la hora de examinar el dolor de los animales? Abordaremos este punto en el próximo apartado.

\section{El humANiSMO EgOlÓGICO DE LA FENOMENOLOGÍA Y SUS LÍMITES}

En el apartado anterior establecimos cuáles son las tres contribuciones capitales de la fenomenología a la filosofía. Dijimos que esas contribuciones eran: mundo, consciencia como dotación de sentido e interioridad sintiente, vale decir, pasividad subjetiva; afección egoica. Apuntamos, además, que dichas contribuciones le otorgaban por vez primera a la filosofía la capacidad analítica de abordar fenómenos que estaban fuera del enfoque filosófico previo al surgimiento de la fenomenología. Uno de los mejores ejemplos de esto lo constituye la reflexión fenomenológica sobre el dolor humano. A continuación, revisaremos sintéticamente el enfoque del dolor humano propuesto por la fenomenología. La revisión del tratamiento fenomenológico del dolor humano nos permitirá, asimismo, establecer si el paradigma fenomenológico representa un camino a seguir a la hora de dilucidar la capacidad animal de sentir dolor.

Toda reconstrucción del enfoque fenomenológico del dolor debe comenzar por hacer mención al trabajo más decisivo alguna vez escrito sobre filosofía del dolor. Nos referimos, evidentemente, al libro del fenomenólogo holandés Frederik Buytendijk (1965); “Teoría del dolor”.

Buytendijk (1965) concibe el dolor como una amenaza no destructiva al ego. En virtud de la operatividad diferencial del fenómeno del dolor es que se explica, a su vez, la reacción humana privativa relativa al dolor, a saber: el estado de alienación. Buytendijk (1965) muestra así que, en el estado de dolor, el hombre no siente su cuerpo como lo hace habitualmente: el dolor está en mí, pero no forma parte de mí. Esta alienación intra-corporal se expresa a través de la manifestación fenomenológicamente más característica del dolor: la localización. A diferencia de todos los demás tipos de afección posible 
(sufrimiento, rabia, fatiga, etc.), el dolor se distingue fenomenológicamente por la localización corporal o concentración estesiológica del malestar. Más aún: Buytendijk (1965) considera que es precisamente gracias al fenómeno de la localización que el hombre puede tomar consciencia de su dolor. La amenaza del dolor se revela como no destructiva en tanto ella aparece restringida a una zona del cuerpo (por lo que no apunta a la totalidad de la integridad subjetiva (Buytendijk, 1965).

Debe destacarse que la sensación de alienación desencadenada por la amenaza no destructiva del dolor introduce una serie de cambios a nivel del cuerpo vivido. La primera modificación característica del dolor en el ámbito corporal es la fijación del horizonte de movimiento: en todo dolor el horizonte del movimiento se subordina a las posibilidades de desplazamiento en relación a la zona adolorida (Leder 1990). De aquí que el dolor desencadene un movimiento centrípeto, vale decir que el dolor contrae la disposición del cuerpo en el espacio hacia la subjetividad: en el dolor, el cuerpo trata de refugiarse en sí mismo.

Sin embargo, el dolor provoca también un movimiento centrífugo: la vivencia del dolor incita a encontrar una nueva disposición hacia el mundo para tolerar o para sobreponerse a él. Todo dolor implica una acción como reacción frente a la sensación desagradable: puede tratarse aquí de un movimiento deliberado (estiramiento) o de un reflejo de acomodación, es decir, de un movimiento que apunta a encontrar una nueva disposición corporal para hacer frente al dolor (como en el caso del dolor crónico) (Leder 1990).

Al mismo tiempo, el dolor implica modificaciones en el ámbito de la temporalidad del cuerpo vivido (Leder 1985). La experiencia del dolor fija el tiempo en el presente: durante el dolor no experimentamos más que el instante doloroso, la vivencia del dolor parece suspendida a tal punto que nuestro horizonte temporal no puede sobreponerse a la sensación dolorosa y nuestra memoria no tiene otro contenido que el momento de la lesión. No obstante, como resultado de la fijación en el presente, el individuo intenta evadirse a través de la nostalgia del estado pre-doloroso (la consciencia escapa así bacia el pasado). Asimismo -y al mismo tiempo-, el dolor implica una proyección del individuo hacia el futuro; buscando refugio en la esperanza del alivio (Leder 1985).

Cabe destacar que los cambios del dolor modifican también la forma de aparición del cuerpo vivido. El psiquiatra norteamericano Drew Leder (1990) ha mostrado de manera admirable que el cuerpo vivido se distingue 
específicamente del cuerpo orgánico gracias a su capacidad de desaparecer. Las dos grandes formas de manifestación del cuerpo vivido tienen como precondición la aparición vía desaparición: el cuerpo vivido puede desenvolverse como sedimento de motivaciones sensori-kinestésicos solamente dejando esta función en la esfera de lo implícito, mientras que la aparición del cuerpo vivido en tanto herramienta práctico-disposicional se vuelve posible manteniendo esta condición gracias a la figura de la virtualidad operativa (Leder 1990).

¿Cómo altera el dolor la aparición del cuerpo vivido? La modificación de la aparición del cuerpo vivido durante el dolor se lleva a cabo mediante dos movimientos. En primer lugar, el dolor concentra las motivaciones sensori-kinestésicas alrededor de la parte adolorida y, volviendo explícita la condición de sedimento sensorial del cuerpo vivido, el dolor hace aparecer a la corporeidad en relación a la hipersensibilización de una región orgánica del cuerpo (Leder 1990).

Asimismo, el dolor impide la aparición del cuerpo vivido como herramienta práctico-disposicional, en tanto el dolor desata un movimiento de coerción cinestésico inmanente: el mundo deja de ser la referencia de la disposición corporal y el cuerpo vivido aparece así en relación a la opresión física que el dolor desencadena. Es a causa de estos dos movimientos en el ámbito de la corporeidad que toda vivencia de dolor conlleva un llamado hermenéutico: las nuevas formas de aparición del cuerpo vivido que derivan del dolor crean la necesidad de una explicitación en relación a dichos cambios (Leder 1990).

Podemos ver que la descripción fenomenológica del dolor se ha concentrado en la cuestión de la alienación resultante de la amenaza no destructiva que el dolor implica para el ego (y de sus expresiones en la esfera del cuerpo vivido). No obstante, todo estado de alienación supone una proyección personal o resonancia subjetiva: “¿Podría alguien pretender que mi dolor de muelas es el conjunto de movimientos que lo expresan y que lo que llama dolor mi conciencia es solo engañosa apariencia? Nadie, sin duda; el dolor no es dolor mientras no lo padece por dentro una consciencia. Como desde fuera no puede sufrirlo espectador alguno, su realidad íntegra, psicológicamente considerada, por lo mismo, su cualidad de dolor, se agota en la fase subjetiva”, escribirá el filósofo chileno, Jorge Millas (2012, pp. 59-60), a la hora de mostrar que toda aproximación fenomenológica al dolor supone destacar la repercusión subjetiva del dolor. 
Es en esta dualidad -alienación como repercusión subjetiva- donde la fenomenología encuentra el segundo aspecto esencial del dolor humano. El fenómeno del dolor, al mismo tiempo (y a pesar de la alineación), implica siempre un sentimiento de auto-identificación como lo ha mostrado magistralmente Ortega y Gasset:

nuestros dolores, que son una de las cosas que se encuentran en el mundo de cada cual o subjetivo, tienen una dimensión positiva en virtud de la cual sentimos por ellos algo así como un afecto -al mismo tiempo que nos están exasperando-, esa como difusa pero cálida actitud que sentimos hacia todo lo auténticamente nuestro. Y es que mientras nos duele nos está, en efecto, siendo íntimo. ¿Cómo no va a ser así, si en el dolor soy siempre yo quien me duelo a mí mismo? (1964, pp. 175-176, cursivas del autor).

En este sentido, la fenomenología muestra que el malestar inherente a la vivencia del dolor se construye a partir de la conjunción e íntima convivencia de alienación y auto-identificación. ¿Cuál es, entonces, el sentido de esta particular conjunción?

Desde un punto de vista teórico, la sola experiencia que fusiona los opuestos -alienación y auto-identificación- es la experiencia del límite. En el límite estoy "aquí" y, al mismo tiempo, esto "más allá”, ya que, como escribiera Karl Jaspers (1986, p. 423): "la palabra límite significa (...) ser aún inmanencia e indicar ya la trascendencia".

La experiencia de la que habla Jaspers muestra exactamente lo que ocurre en el dolor: la auto-identificación en el dolor da cuenta de la parte más propia de mi mismidad (del "aquí y ahora de mi cuerpo"), mientras que la alienación nos habla del estado de virtualidad en el que el cuerpo se encuentra ya más allá de su inmediatez. Esta dualidad pone de relieve el significado fundamental del fenómeno del dolor, a saber: el sentido del dolor es la experiencia del límite de mi propio cuerpo (Mujica, 2018a). En cada dolor hacemos la experiencia del límite de nuestro cuerpo, es decir, que tocamos los límites que nuestro esquema corporal ha sistematizado como respuesta a los estímulos senso-kinestésicos, a la vez que nos encontramos con los límites de nuestro "Yo-puedo" y de nuestra capacidad de resistencia frente al mundo (Mujica, 2018b).

La reconstrucción esquemática de los presupuestos fenomenológicos del tratamiento del dolor (alienación, auto-identificación, etc.) nos ha permitido, no sólo acceder al sentido de la experiencia del dolor, sino a sopesar nuevamente la contribución filosófica de la fenomenología: la descripción esencial 
del dolor humano sólo se vuelve posible cuando se cuenta con herramientas analíticas que permiten explicar, por ejemplo, elementos como el "llamado hermenéutico" en el dolor (consciencia como atribución de sentido), la autoidentificación en la vivencia del dolor (interioridad, pasividad) o la caracterización del dolor como amenaza (mundo como condición de posibilidad de toda amenaza). Sin embargo, esta descripción también señala límites: no queda claro hasta qué punto las categorías ego (interioridad, afección), sentido y mundo resultan operativas en el caso de los animales. Más aún, como veremos a continuación, ego, sentido y mundo son concebidos por la fenomenología como privativamente humanos. De lo anterior se desprende que, por lo menos en el marco del paradigma fenomenológico, el dolor sería patrimonio humano (y no animal).

Pero determinar si la fenomenología sigue siendo un enfoque válido para abordar el dolor animal supone explicitar teóricamente los conceptos que han permitido una descripción fenomenológica del dolor humano: solo a través de este ejercicio analítico será posible establecer si los conceptos de ego, mundo y sentido tienen un alcance analítico extrapolable a la consistencia diferencial de la animalidad.

Veamos, en primer lugar, si el concepto fenomenológico de mundo es susceptible de utilización en el caso de los animales. Todo esclarecimiento de la categoría mundo en fenomenología debe comenzar por el análisis del mundo propuesto por Heidegger (2002, \&15-19)) en "Ser y tiempo" (análisis retomado en "Conceptos fundamentales de la metafísica" (Heidegger, 2007, \&42-63).

Heidegger (2002) concibe el mundo como una estructura de significatividad. Esta condición del mundo no remite a la capacidad subjetiva de mentar fines; ni a la entidad de las cosas en cuanto tal. El mundo, según Heidegger (2002), se revela como una trama integral de sentido en virtud de la especificidad de su estructura diferencial, a saber: la remisión.

La experiencia de la más simple cotidianidad da cuenta que el estar del hombre en el mundo se distingue por el dejarse conducir a partir de la estructura de las remisiones que el mundo pone a nuestra disposición. En este sentido, cada acontecimiento o manifestación -cada ente- supone siempre una remisión: en la donación misma de un objeto en su aparecer mundano está supuesta su condición de "para-qué". Es así que cada ente puede aparecer en el mundo como útil o como obstáculo, como indicación explícita o como indiferencia: el mundo se revela fundamentalmente, entonces, como signo (Heidegger, 2002, \&17). Pero, ¿como signo de qué?: como signo de lo 
que está a la mano. Por eso Heidegger dirá que un signo es lo que indica a qué atenerse en el trato intramundano con el mundo. Justamente por esto el gran rendimiento del signo es garantizar el trato con el complejo de lo a la mano. Es por esto que cuando decimos que el mundo es una estructura de significatividad, debe tomarse la expresión con el máximo rigor, ya que sólo gracias a la trama de signos (y de remisiones asociadas a éstos) aparece el mundo en su condición diferencial, a saber: ser una trama integral de señales que nos indica cómo se accede y se trata con lo dado (Heidegger, 2002, \&19).

Al margen de la complejidad filosófica de este problema -y del tratamiento heideggeriano del mismo-, debe destacarse que la experiencia del mundo como plexo significativo de remisiones no se constituye como una adquisición del análisis filosófico. El mundo opera como estructura de significaciones en la más simple cotidianidad: el campesino sabe que la chumbera se cultiva por la madrugada (el rocío matinal impide el espolvoreo de espinas), de igual forma que puede anticipar la lluvia de la siembra gracias a la señal del viento. Todo acontecer mundano es un "respecto de", y ese "respecto de" no es otra cosa que un signo de: "aquello con lo que un trato es posible o necesario para el modo de ser de lo ente" (Heidegger, 2007, p. 247).

Heidegger (2007, p. 247) sintetiza su concepto de mundo a través de la siguiente frase: "Mundo (...) es lo ente respectivamente accesible y lo ente tratable, lo que es accesible". Mundo es, entonces, lo que permite el acceso a lo ente; al sistema de referencias que los útiles en su "para-qué" ponen a nuestra disposición. La cuestión es, entonces, saber si los animales hacen o no la experiencia del mundo.

El hombre tiene mundo porque puede disponer; las cosas aparecen para él a partir de un para qué, de un "respecto de" o, como dice Heidegger (2002, \&15-19), el ente se revela al hombre en cuanto ente bajo la forma de útiles: es la disponibilidad y la referencia contextual de lo ente como útil lo que determina la condición de mundo como accesibilidad. Pero, ¿puede el animal disponer?

Heidegger (2007) intentará responder a esta cuestión a partir de une reflexión sobre la esencia de la forma de vida de la animalidad. Heidegger (2007) encuentra la estructura esencial de la vida animal en la noción de organismo. Puede verse ya que, a nivel estructural, la noción de organismo contrasta específicamente con la capacidad de disponer de lo útil propia del hombre: "Los órganos no están presentes en el animal, sino que están al servicio de sus capacidades. Este estar al servicio hay que tomarlo con todo rigor (...) los 
órganos están totalmente sujetos a este servicio (...) Lo útil, por el contrario, nunca es servicial” (Heidegger, 2007, p. 283, cursivas del autor).

Pero si el organismo no puede disponer, ¿cuál es, entonces su operación diferencial? En tanto el animal está al servicio de sus órganos, la conducta del animal: "sólo es posible en función del estar cautivado en sí del animal” (Heidegger, 2007, p. 291, cursivas del autor). Al margen de la capacidad hipotética que los órganos del animal entregan, su conducta no es nunca un disponer, sino, más bien, un estar absorbido por sí mismo. Heidegger denomina este sello de la conducta animal perturbamiento: "El estar-consigo especificamente animal, que no tiene nada que ver con la mismidad del hombre que se comporta en tanto que persona, este estar cautivado en sí del animal, en el que es posible toda conducta, lo designamos perturbamiento" (Heidegger, 2007, p. 291, cursivas del autor).

Si el hombre está dispuesto, el animal está perturbado: esa es su condición esencial a juicio de Heidegger. Ahora bien, ¿qué implica esto en el contexto de nuestra investigación? Pues básicamente que su condición de absorción, de perturbamiento esencial, le impide toda forma de mundo: "El perturbamiento es la condición de posibilidad de que el animal, conforme a su esencia, se conduzca en un medio circundante, pero jamás en un mundo" (Heidegger, 2007, p. 291, cursivas del autor).

Estamos ahora en condiciones de comprender en toda su radicalidad la hipótesis con la que Heidegger (2007, \&42) comienza su análisis de la esencia de la animalidad, a saber: “el animal es pobre de mundo”. A primera vista, a diferencia de la piedra, pareciera que el animal tiene algún grado de acceso al mundo: "El lagarto no se limita a aparecer sobre la piedra calentada al sol. Se ha buscado la piedra (...) Se tumba al sol. Así lo decimos, aunque es dudoso si en ello se comporta como nosotros cuando estamos tumbados al sol, si el sol le es accesible en cuanto sol” (Heidegger, 2007, p. 248; cursivas del autor). Sin embargo, la determinación de la esencia de la animalidad como perturbamiento limita al animal a conducirse exclusivamente a partir de determinados estímulos privativos de su medio; sustrayéndole así toda posibilidad de acceso al mundo: "del perturbamiento forma parte, como un momento esencial, la sustracción del mundo" (Heidegger, 2007, p. 327; cursivas del autor).

La reconstrucción del concepto fenomenológico de mundo, así como el tratamiento de la esencia de la animalidad en el pensamiento de Heidegger, nos ha permitido llegar a una primera conclusión a la hora de evaluar la atingencia del paradigma fenomenológico como método de abordaje del dolor animal, a saber: el animal no tiene mundo. Esto implica que el animal no 
puede otorgar a la sensación dolorosa su condición de amenaza: el animal no tendría, en este sentido, la capacidad moduladora necesaria para concebir el dolor en cuanto dolor. No obstante, los análisis de Heidegger también nos permitirán determinar si la categoría fenomenológica sentido es aplicable a la realidad del dolor animal.

El hecho de que el mundo sea tratado por Heidegger en tanto plexo de remisiones, signos y referencias da cuenta de la inseparabilidad constitutiva de sentido y mundo. El mundo se revela como una estructura de significaciones precisamente porque su operación esencial es mentar, indicar, referir, atribuir. Vimos en el apartado anterior cómo la fenomenología identificaba la operación de sentido con una referencia de inteligibilidad diferencial para con el fenómeno mentado. Si el hacer del hombre es siempre un referir -una donación de sentido, veamos ahora en qué consiste el hacer del animal a partir de su particular condición.

El análisis de Heidegger de la esencia de la animalidad sigue siendo de utilidad en este contexto, porque él también muestra cuáles son los alcances de la conducta del animal a partir de la revelación de su condición fundamental de perturbamiento:

"El hacer no es un orientarse constatador hacia cosas objetivamente presentes, sino una conducta. El hacer es una conducta. Con ello no se niega que de la conducta forme parte algo así como un "hacia" el aroma y la miel, una referencia a..., pero no es un orientarse constatador hacia ello o, dicho más exactamente, no es una percepción de la miel en tanto que algo presente, sino un peculiar perturbamiento" (Heidegger, 2007, p. 295; cursivas del autor).

A pesar de que Heidegger concede que en toda conducta animal puede distinguirse una especie de proto-referencia, esto no implica una donación de sentido a la conducta animal, en tanto la conducta animal se revela como determinada a priori como consecuencia del perturbamiento característico de la esencia de la animalidad: "La conducta animal nunca se refiere-como podría parecernos- a cosas presentes y a su acumulación, sino que rodea a sí mismo de un anillo de desinbibición, en el que está prefigurado qué es lo que su conducta puede encontrar como motivo." (Heidegger, 2007, p. 309; cursivas del autor).

La proto-referencia de la conducta animal no puede ser considerada como una variante de donación de sentido, y esto en virtud de dos razones, a saber: la conducta no es, en estricto rigor, una referencia o una mención (puesto que ella está predefinida) y, justamente por eso, el referir del animal no constitu- 
ye una forma de atribución de inteligibilidad. La razón es simple: sus formas de atribución están predeterminadas en virtud del anillo de desinhibición que la pertenencia a determinada especie establece como forma de perturbamiento diferencial y como posibilidad de conducta definida.

Nuestro análisis preliminar de los presupuestos que permitieron a la fenomenología el tratamiento filosófico del dolor humano se han revelado mezquinos a la hora de abordar el dolor animal: la fenomenología invita a concluir que el animal no tiene mundo y que su conducta no se enmarca dentro de la categoría sentido. Dado lo anterior, la capacidad animal de sentir dolor se vería fuertemente restringida en virtud de su incapacidad de acceder a remisiones mundanas (imposibilidad de registrar la localización del dolor), así como de atribuir sentido al dolor (lo que denominamos más arriba como "llamado hermenéutico"). Veamos si las herramientas conceptuales de la fenomenología consideran la pasividad egoica como un componente de la realidad animal.

El más sistemático de los análisis fenomenológicos sobre la interioridad -entre otras cosas, por ser el único que considera a los animales-, fue llevado a cabo por Ortega y Gasset (1964) en su libro El hombre y la gente.

En su tratamiento de la realidad filosófica radical -la vida- Ortega subrayará que la vida es siempre una hacer (Ortega y Gasset, 1964). No obstante, este hacer puede adoptar dos formas fundamentales: puede tratarse de un hacer externo o mecánico (impuesto por coacción o por convención), y puede tratarse de un hacer auténtico, íntimo (resultante de una determinada convicción o necesidad vital) (Ortega y Gasset, 1964). Dado lo anterior, Ortega asociará cada forma de hacer con una disposición constitutiva de la vida humana: en tanto la primera disposición es atribuible a la fuerza de factores externos (Estado, gente, sociedad), Ortega la denominará alteración. En el hacer externo (en el saludo, en el estadio) el hombre está fundamentalmente alterado. Por el contrario, en el hacer auténtico (en su íntima deliberación), el hombre está recogido, meditando o, como dice Ortega, ensimismado (Ortega y Gasset, 1964).

El descubrimiento de esta dualidad privativa de la vida humana le permitirá a Ortega trazar la diferencia constitutiva con el animal:

En ninguna parte advertimos que la posibilidad de meditar es, en efecto, el atributo esencial del hombre mejor que en el Jardín Zoológico, delante de la jaula de nuestros primos, los monos (...) Si sabemos permanecer un rato quietos contemplando pasivamente la escena simiesca, pronto destacará de ella, 
como espontáneamente, un rasgo que llega a nosotros como un rayo de luz (...) son los objetos y acaecimientos del contorno quienes gobiernan la vida del animal, le traen y le llevan como una marioneta. Él no rige su existencia, no vive desde sí mismo, sino que está siempre atento a lo que pasa fuera de él, a lo otro que él. Nuestro vocablo otro no es sino el latino alter. Decir, pues, que el animal no vive desde sí mismo sino desde lo otro, traído y llevado y tiranizado por lo otro, equivale a decir que el animal vive siempre alterado, enajenado, que su vida es constitutiva alteración (Ortega y Gasset, 1964, p. 83; cursivas del autor).

A diferencia del hombre, Ortega muestra que el animal se caracteriza, desde el punto de vista fenomenológico, por no poder ensimismarse; por carecer de interioridad: "El animal es pura alteración. No puede ensimismarse. Por eso, cuando las cosas dejan de amenazarle o acariciarle; cuando le permiten una vacación; en suma, cuando deja de moverle y manejarle lo otro que él, el pobre animal tiene que dejar virtualmente de existir, esto es: se duerme" (Ortega y Gasset, 1964, p. 85; cursivas del autor). Vemos así que el animal, a juicio de la fenomenología, no sólo carece de mundo y de sentido; carece asimismo de interioridad. Naturalmente que esta conclusión implica que el dolor no formaría parte de la realidad animal: los animales no son capaces de sentido, no tienen mundo, ni tienen interioridad; se encontrarían, por ende, incapacitados de sentir dolor.

Nuestra tentativa de aprovechar los rendimientos descriptivos de la fenomenología para abordar el dolor animal ha entregado, hasta ahora, sólo resultados negativos. Aunque la fenomenología descubrió en las categorías mundo, sentido e interioridad las herramientas conceptuales para acceder filosóficamente al dolor humano, ella se revela -desde el punto de vista teórico- como un humanismo egológico (en la medida en que el dolor es consecuencia de la estructura de la subjetividad humana y de su constitución diferencial). No obstante, esta posición se contrapone con la evidencia indesmentible de la reacción animal frente al dolor (así como al aprendizaje animal de conductas para evitarlo y al desarrollo de una gestualidad distintiva asociada al dolor). La cuestión no es aquí si el fenómeno del dolor es condición de posibilidad de un cierto aparataje teórico que permitiría describirlo: nuestra hipótesis es que lo animales sienten dolor con independencia de si esa experiencia se condice o no con los presupuestos teóricos del análisis fenomenológico del dolor humano. Concluiremos este trabajo proponiendo algunos lineamientos para el desarrollo de una filosofía del dolor animal. 


\section{Pasos hacia una filosofía del dolor ANIMAL}

El objetivo del apartado final de este trabajo es ofrecer nociones para el desarrollo de una filosofía del dolor animal. No se discutirá acá sobre la cuestión moral ni sobre la justicia y/o justificación del dolor animal. En contraste con el supuesto deontológico que atraviesa la reflexión filosófica sobre el dolor animal (Cavalieri, 2001, Francione, 2008; Garner, 2013; Regan, 2004; Singer, 1975) se trata aquí de una cuestión más básica, más fundamental, a saber: establecer si los animales pueden sentir dolor. No obstante, el apartado anterior de este trabajo concluyó con una aporía: explicitamos la impertinencia de las herramientas fenomenológicas a la hora de describir el dolor animal, pero, al mismo tiempo, el dolor animal aparece como una evidencia indesmentible. La cuestión no es, entonces, si el dolor animal es posible o no, sino cuál es la manera filosófica apropiada de abordarlo para dar cuenta de su unicidad. A continuación, esbozaremos criterios teóricos que permitan enfocar la realidad del dolor animal en su especificidad diferencial.

Por cierto, que la crítica a la pertinencia de la fenomenología como paradigma de enfoque del dolor animal no implica renunciar a las intuiciones que ella pueda aportar a la hora de abordar la cuestión del dolor en los animales. En este sentido, la caracterización del dolor llevada a cabo por Buytendijk puede servirnos, no como caracterización en cuanto tal, sino alpensar su revés. Dijimos que Buytendijk (1965) considera el dolor humano como una amenaza no destructiva para el ego. Fiel a las consecuencias teóricas del humanismo egológico de la fenomenología, Buytendijk niega a los animales la capacidad de experimentar dolor en sentido estricto: "Un animal nunca logrará adquirir conciencia del yo por más que experimente muchas impresiones dolorosas, ya que carece de la capacidad de asociarlas con su propia experiencia en el ámbito existencial. Un animal puede, por cierto, sufrir daño, pero no en su propia unidad psicofísica” (Buytendijk 1965, p. 147).

Para Buytendijk (1965) el animal no puede experimentar dolor porque no tiene la capacidad de sentir amenazada su integridad (ya que no tiene noción de ella). El hombre, por el contrario, puede sentir el dolor como una amenaza no destructiva, no solo porque su interioridad le entrega la certeza de poseer una integridad subjetiva, sino porque ella colabora asimismo a modular el dolor: todo hombre sabe cuándo el dolor deviene una amenaza destructiva (es decir, cuando se trata de una experiencia que ya no es solamente dolor). Buytendijk (1965, p. 29) denomina esta situación "abandono inconsciente del ser humano al dolor". 
Lo interesante a destacar en nuestro contexto es que Buytendijk reconoce una experiencia análoga en el caso de los animales: "Este abandonarse inconsciente del ser humano al dolor tiene por resultado directo un divorcio del yo y su cuerpo. Aun el animal, mucho menos protegido contra el dolor que nosotros, conoce esta experiencia y reacciona a ella de una manera elemental. Ya en un desposeimiento de sí misma, la criatura es presa de las garras del dolor. Los gritos salvajes parecen expresar su deseo de abandonar su cuerpo al dolor. Esto constituye una reacción refleja difusa dolor, surgida desde el centro inconmovible del dolor. Cuando experimenta dolor la criatura tiende a disociarse de su cuerpo" (Buytendijk 1965, p. 29-30, cursivas del autor).

Vemos así que una observación cuidadosa al dolor animal reconoce en él un patrón. Buytendijk muestra que, frente al dolor, el animal parece disociado de su cuerpo; desposeído de sí mismo. Frente a la capacidad humana de modular la sensación dolorosa y de concebirla como amenaza no destructiva, el dolor animal parece consistir en una amenaza -no sólo destructiva para el animal-, sino que total: la mínima observación del dolor animal permite comprender que el dolor es una condición completamente des-estabilizante para el animal (no así para el hombre).

Un primer criterio para avanzar hacia una filosofía del dolor animal es, entonces, concebir el dolor animal como una amenaza destructiva y total para el animal. Más aún, el dolor animal se corresponde con una sensación no sólo total, destructiva; sino que sin referencias. El análisis de Heidegger (2007) sobre el perturbamiento como condición esencial del animal permite describir el dolor animal desde el punto de vista filosófico como una amenaza que no forma parte del registro fundamental del animal. Resulta fácil concluir así que el animal es indiferente al dolor dado que dicha sensación no formaría parte de su registro vital. Nos parece que la conclusión necesaria es justamente la contraria: la incapacidad de interpretar vuelve a la sensación dolorosa en el animal un desafío aún más traumático que en el caso del hombre.

Llegamos así a una primera caracterización provisional del dolor animal. Se trata, en su caso, de una sensación total (invade completamente su existencia), destructiva (no es modulable, ergo vivida como un peligro para su subsistencia), e ininteligible -en tanto la sensación dolorosa no forma parte de su registro vital, ella no puede ser procesada (revelándose, para el animal, como pura inminencia).

Sin embargo, esta caracterización provisional del dolor animal debe ser confrontada con una objeción de carácter epistemológico, a saber: ella se basa en una atribución humana. Asimismo, nosotros "sabemos" que el animal 
sufre en virtud de una atribución hecha desde la consciencia moral y, sobre todo, desde una proyección cinestésico-estesiológica de tipo analógica. No obstante lo anterior, el animal nada sabe de lo que hemos dicho. Más aún: el animal nada sabe de su dolor. Es una consecuencia necesaria de la ininteligibilidad de su registro. El animal padece el dolor sin poder interpretarlo, sin saber que lo padece (ni lo que padece). Se trata así de un padecimiento total, ergo anónimo. El filósofo suizo Luc-François Dumas (2015) dirá que el padecer animal se revela como un "sufrimiento inhabitado". La fórmula es justa a pesar de lo paradójica que pueda resultar: pareciera que más que padecerlo, el animal se ve poseído por su dolor. Y como todo ser poseso, el animal se ve impedido de apropiarse de lo que padece: por eso el dolor animal parece no tener medida.

Pero no se trata solo de un dolor sin medida en el caso del animal, se trata -asimismo- de un dolor sin sedimentación. El animal pareciera estar relativamente al margen del proceso de sedimentación corporal que deriva del dolor (Mujica, 2018a). Esto hace que el animal esté también al margen de la posibilidad del trauma como lo había visto Buytendijk: "La explicación del trauma radica en el hecho de que la aflicción dolorosa de un sujeto se vincula con la experiencia de que es él (y no otro) el que ha sido afectado. El individuo debe estar entonces "en posición de sí mismo". Esto no ocurre (...) entre los animales" (Buytendijk 1965, p. 149; cursivas y comillas del autor). De aquí que todo dolor sea, para el animal, algo desconocido, incomprensible, siempre un novel encuentro con la inminencia1. De ahí la justeza de la caracterización

1. Urge en torno a este tema conectar los desarrollos de la biología evolutiva con el enfoque fenomenológico. La fenomenología ha mostrado, por una parte, la relación constitutiva entre identidad, memoria y pasividad (Kühn, 1998). Parecería, así, desde un punto de vista teórico, que la memoria aparece como un requisito del dolor. La cuestión es entonces, dada la incapacidad de memoria entre peces y crustáceos (Bateson, 1993), determinar si es posible hablar de sensación dolorosa en el caso de tales animales. No obstante, este dato se contrapondría con una reacción genérica que tienen las plantas frente a su mutilación (Bateson, 1998). Falta aclarar si existe un gradiente evolutivo lineal en términos de sistema nervioso en lo que respecta a la posibilidad de la sensación dolorosa. En esto neurociencia y fenomenología tampoco logran un acuerdo total: mientras la neurología muestra que el bebé requiere de seis meses para distinguir el dolor de otras formas de malestar (hambre, fatiga, temor, etc. (Marchand, 2009), la fenomenología muestra que el desarrollo del ego -ergo de la capacidad de sentir-, tiene como condición de posibilidad la constitución de un referente primordial de atribución de sentido (referente que Husserl encuentra en la madre como traspaso de la capacidad de sentir a través de la progresiva diferenciación de la carne en el vientre materno (Lee, 1993). 
que propone Buytendijk sobre la reacción animal al dolor: "la reacción de retroceso del animal y la emoción de temor creada en él se producen por haber reconocido una situación en la que previamente experimentó dolor, y no por la consecuencia de su acción presente, es decir, el dolor" (Buytendijk 1965, p. 106).

Sin embargo, la caracterización de un dolor no sedimentable en el caso del animal se contrapone con la evidencia del aprendizaje de conductas motivadas por el dolor y el padecer animales. Esto invita a pensar que la negación de la categoría sentido a los animales por parte de la fenomenología requiere de una revisión radical:

Es más difícil precisar la relación del sentido con respecto a los animales. Las discusiones teórico-técnicas al respecto no permiten concluir algo decisivo, ya que cuando observamos a los animales, lo hacemos desde un mundo pleno de sentido que no permite tomar la perspectiva idéntica a la del animal para imaginar cómo ordenar la perspectiva que ellos se hacen sobre el mundo. Mucho habla a favor de que los animales poseen una especie de proto sentido, sobre todo cuando se observa la fluidez y elegancia con la que los animales se mueven de una situación a otra, y no sólo cuando se los observa de manera esporádica resolviendo situaciones muy particulares. Entonces se inclina uno a pensar que los animales contemplan el espacio pleno de sentido, y en relación a un antes y un después. (Luhmann, 2002, p. 177, cursivas del autor).

Pero no solo debe revisarse la posibilidad de un gradiente de sentido en el caso de los animales a partir de la evidencia que aporta su conducta frente al dolor, sino la pérdida del mismo en el caso del dolor animal. En tanto el dolor parece no formar parte del registro vital del animal, él se revela -no como la experiencia del límite del cuerpo (como en el caso del hombre)-, sino como una irrupción en el equilibrio de su condición: el dolor parece impedir totalmente el desenvolvimiento normal de la conducta del animal. En este sentido, si el animal parece mostrar ciertos atisbos de sentido a la hora de volver a enfrentar el dolor, parece perderlo absolutamente cuando este acaece. Se trata así de un padecer mucho más profundo que el del hombre. Si en el dolor el hombre se ve confrontado explícitamente al límite de su propio cuerpo, el animal ve amenazada, en el dolor, la totalidad de su equilibrio. No parece haber sentir más radical: si en el dolor humano el mundo aparece como una amenaza, el ambiente vital del animal es atravesado por el dolor (al punto que todo el orden que el animal había articulado parece destruido). Se trata entonces de una pura negatividad, negatividad casi imposible de procesar, he 
ahí la indefensión radical del animal en su dolor y la correlativa pérdida de las formas de proto-sentido que se habían ganado. Tal vez esta caracterización del estado animal frente al dolor nos sirva, a su vez, para recuperar el sentido ínclito de la palabra animal, a saber: "lo que tiene alma" (Bateson, 1993).

\section{BIBLIOGRAFÍA}

Bateson, G. (1993). Espiritu y naturaleza. Buenos Aires, Amorrortu.

Bateson, G. (1998). Pasos hacia una ecología de la mente. Buenos Aires, Lumen.

Bourgeois, B. (1995). La philosophie allemande classique. Paris, PUF.

Buytendyjk, F. (1965). Teoría del dolor. Buenos Aires, Troquel.

Cavalieri, P. (2001). The Animal Question: Why Nonbuman Animals Deserve Human Rights. New York, Oxford University Press.

De Waelhens, A. (1953). Phénoménologie et vérité. Essai sur l'évolution de l'idée de vérité chez Husserl et Heidegger. Paris, PUF.

Dumas, L. F. (Productor) (2015). 10 Philosophie -L'animal-Connaissance et affectivité https://www.youtube.com/watch? $=\mathrm{CvxPvK} 7 \mathrm{tBF} 4$.

Francione, G. (2008). Animals As Persons: Essays on the Abolition of Animal Exploitation. Nueva York, Columbia University Press.

Frank, M. (1997). Unendliche Annäherung : Die Anfänge der philosophischen Frübromantik. Frankfurt, Suhrkamp.

GadAmer, H. G. (1993). Verdad y método. Salamanca, Sígueme.

Garner, R. (2013). A Theory of Justice for Animals. Oxford, Oxford University Press.

Heidegger, M. (2002). Ser y tiempo. Santiago, Editorial Universitaria.

Heidegger, M. (2007). Los conceptos fundamentales de la metafísica. Mundo, finitud, soledad. Madrid, Alianza editorial.

Henry, M. (2001). Incarnation. Une philosophie de la chair. Paris, Seuil.

Housset, E. (2008). Husserl et l'énigme du monde. Paris, Seuil.

Husserl, E. (1982). Idea de la fenomenología. Cinco lecciones. México, FCE.

Husserl, E. (1995). Ideas relativas a una filosofía pura y a una filosofía fenomenológica. México, FCE.

Husserl, E. (2004). Meditaciones cartesianas. México, FCE.

Husserl, E. (1959). Recherches logiques. 1, Prolégomènes à la logique pure. Paris, PUF.

Husserl, E. (1998). De la synthèse passive. Grenoble, Jérôme Million.

Jaspers, K. (1986). Philosophie : orientation dans le monde, éclairement de l'existence, métaphysique. Paris, Springer-Verlag.

KüHN, R. (1998). Husserls Begriff der Passivität: Zur Kritik der passiven Synthesis in der Genetischen Phänomenologie. Freiburg, Alber. 
Leder, D. (1985). “Towards a Phenomenology of Pain”. Review of Existential Psychology and Psychiatry 19, pp. 255-266.

Leder, D. (1990). The absent body. Chicago, Chicago University Press.

LeE, N-I. (1993). Edmund Husserls Phänomenologie der Instinkte. Dordrecht, Kluwer.

Levinas, E. (1967). “Intentionnalité et métaphysique”. Découvrant l'existence avec Husserl et Heidegger. Paris, VRIN, pp. 137-144.

Levinas, E. (2004). La teoría fenomenológica de la intuición. Salamanca, Sígueme.

LuHmann, N. (2002). Introducción a la teoría de sistemas. México, Universidad Iberoamericana.

Marchand, S. (2009). La douleur. Comprendre pour soigner. Paris, Elsevier/Masson.

Marcuese, H. (2003). Razón y revolución. Madrid, Alianza.

Merleau-Ponty, M. (2001). Phénoménologie de la perception. Paris, Gallimard.

Millas, J. (2009). Idea de la individualidad. Santiago, UDP.

Montavont, A. (1999). De la passivité dans la phénoménologie de Husserl. Paris, PUF.

Mujica, F. (2018a). Éprouver la limite. Phénoménologie de la douleur (Tesis doctoral). Université Catholique de Louvain, Louvain-La-Neuve.

Mujica, F. (Mayo de 2018b). La filosofía fenomenológica como puerta de acceso al sentido de la experiencia del dolor. En (Velarde) Congreso International Philosophy of pain/ Filosofía del dolor. Conferencia llevada a cabo en la Universidad de Salamanca, Salamanca.

Ortega y Gasset, J. (1963). “Conciencia, objeto y las tres distancias de éste”. En: Obras completas II. Madrid: Revista de Occidente, pp. 61-69.

Ortega y Gasset, J. (1964). "El hombre y la gente”, en: Obras completas. Tomo VII. Madrid, Revista de Occidente, pp. 71-274.

Ortega y Gasset, J. (1966). "Max Scheler. Un embriagado de esencia” Obras completas IV. Madrid: Revista de Occidente, pp. 507-513.

Regan, T. (2004). The Case for Animal Rights, Berkeley, University of California Press.

Romano, C. (2010). Au coeur de la raison, la phénoménologie. Paris, Gallimard.

SARTRE, J.P. (1984). La imaginación. Madrid, Sarpe.

Singer, P. (1975). Animal Liberation: A New Ethics for our Treatment of Animals, New York Review/Random House, Nueva York.

SzILASI, W. (1973). Introducción a la fenomenología de Husserl. Buenos Aires, Amorrortu. 